\title{
Circular RNA profiling distinguishes medulloblastoma groups and shows aberrant RMST overexpression in WNT medulloblastoma
}

\author{
Daniel Rickert ${ }^{1,2,3,4}$. Jasmin Bartl ${ }^{1,2,3,4} \cdot$ Daniel Picard $^{1,2,3,4} \cdot$ Flavia Bernardi $^{5,6} \cdot$ Nan Qin $^{1,2,3,4} \cdot$ Marta Lovino $^{7}$. \\ Stéphanie Puget ${ }^{8} \cdot$ Frauke-Dorothee Meyer $^{1,2,3,4} \cdot$ Idriss Mahoungou Koumba $^{2,3} \cdot$ Thomas Beez $^{9} \cdot$ Pascale Varlet $^{10}$. \\ Christelle Dufour $^{11,12}$. Ute Fischer ${ }^{2,3} \cdot$ Arndt Borkhardt $^{1,2,3} \cdot$ Guido Reifenberger $^{1,2,4}$. Olivier Ayrault ${ }^{5,6}$. \\ Marc Remke ${ }^{1,2,3,4}$ (1)
}

Received: 28 January 2021 / Revised: 25 March 2021 / Accepted: 29 March 2021 / Published online: 17 April 2021

(c) The Author(s) 2021

Medulloblastoma is the most common malignant brain tumor in childhood [7]. Molecular classification into WNT, SHH, Group 3, and Group 4 of medulloblastoma refines surveillance of tumor predisposition syndromes, improves risk stratification to (de-)escalate therapeutic interventions, and sets the stage for targeted therapies [7]. Discrimination between Group 3 and Group 4 medulloblastoma remains challenging with partly inconsistent group annotation using DNA methylation data [1], gene expression profiling [8] or (phospho-)proteomics [3]. Thus, studies are increasingly integrating two or more molecular layers for accurate group assessment of this heterogeneous disease $[1,8]$.

Circular RNAs (circRNA) recently emerged as promising biomarkers with cell type- and developmental stage-specific expression patterns in human tissues and cancers [10]. They are not easily degraded by exonuclease RNase R, are

Daniel Rickert and Jasmin Bartl shared first authors.

Olivier Ayrault and Marc Remke shared last/co-corresponding authors.

Marc Remke

marc.remke@med.uni-duesseldorf.de

1 Division of Pediatric Neuro-Oncogenomics, German Cancer Research Center (DKFZ), Heidelberg, Germany

2 German Cancer Consortium (DKTK), Partner Site Essen/Düsseldorf, Düsseldorf, Germany

3 Department of Pediatric Oncology, Hematology, and Clinical Immunology, University Hospital Düsseldorf and Medical Faculty, Heinrich Heine University, Düsseldorf, Germany

4 Institute of Neuropathology, Düsseldorf and Medical Faculty, University Hospital, Heinrich Heine University, Düsseldorf, Germany

5 Institut Curie, CNRS UMR, INSERM, PSL Research University, Orsay, France long-lived and are widely detected in body fluids [10]. Based on these unique properties, circRNAs may serve as clinically useful diagnostic biomarkers. We developed a bioinformatic approach called "circs", combining three circRNA detection pipelines (find_circ [5], DCC [2], and CIRCexplorer [11]) reducing the false-positive rate compared to single in silico circRNA detection method.

We could detect circRNAs with higher expression in medulloblastoma (Figures S1a and S1b, Table S1), but most circRNAs were significantly lower expressed compared to fetal brain tissue samples as previously reported (Fig. 1a) [10]. In contrast to the previous indications that non-coding RNA expression profiles were not sufficient to distinguish groups [9], unsupervised hierarchical clustering revealed the four core groups using the top 500 most differentially expressed circRNAs in our discovery cohort $(n=38$, Fig. 1b, Table S2), and in our non-overlapping validation cohort $(n=35$; Fig. 1c, Table S3). We confirmed medulloblastoma classification of the discovery cohort based on the previous similarity network fusion (SNF) analysis (Fig. 1d, data

6 CNRS UMR 3347, INSERM U1021, Université Paris Sud, Université Paris-Saclay, Orsay, France

7 Department of Control and Computer Engineering, DAUIN, Politecnico di Torino, Torino, Italy

8 Department of Pediatric Neurosurgery, Necker Hospital, APHP, Université Paris Descartes, Paris, France

9 Medical Faculty, Department of Neurosurgery, Heinrich Heine University, Düsseldorf, Germany

10 Department of Neuropathology, GHU Paris-Neurosciences, Sainte-Anne Hospital, 75014 Paris, France

11 Department of Pediatric and Adolescent Oncology, Gustave Roussy, Villejuif, France

12 INSERM, Molecular Predictors and New Targets in Oncology, University Paris-Saclay, Villejuif, France 


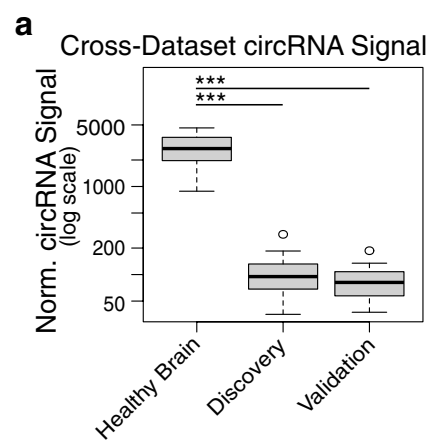

d

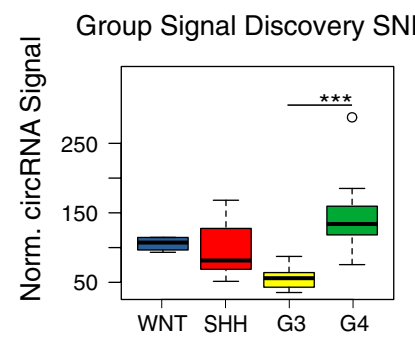

b

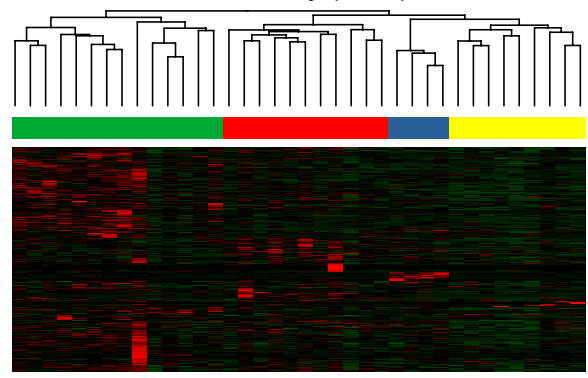

e

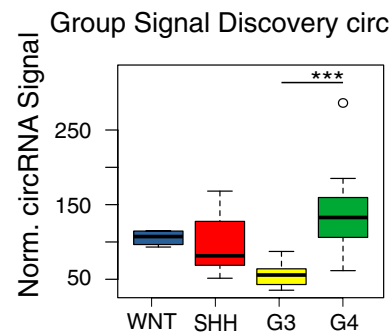

C

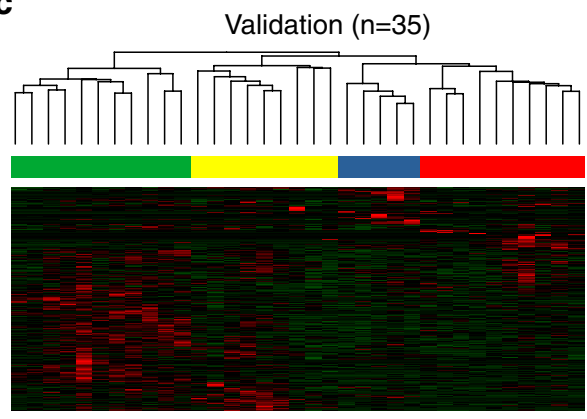

f

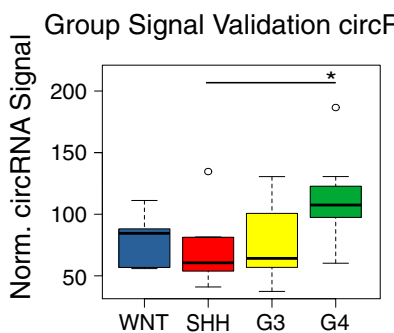

g

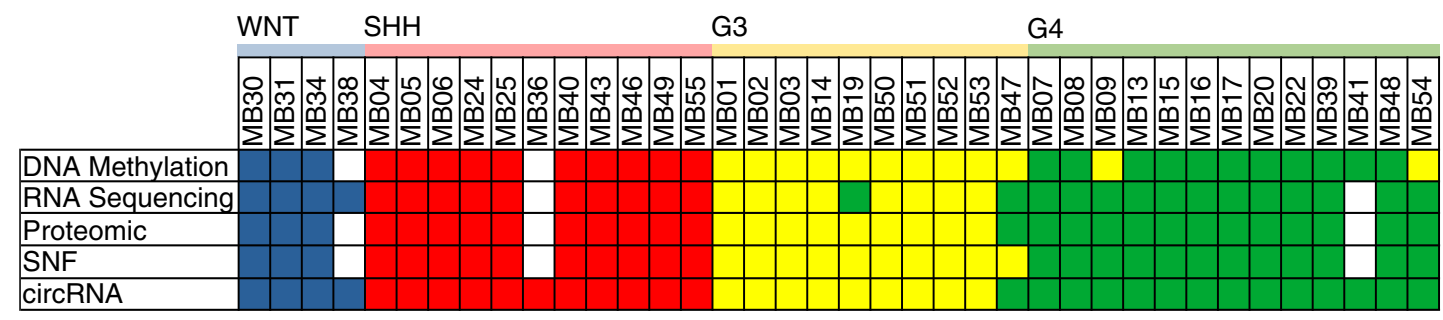

h

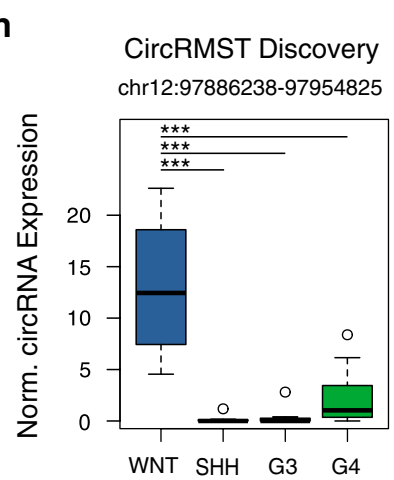

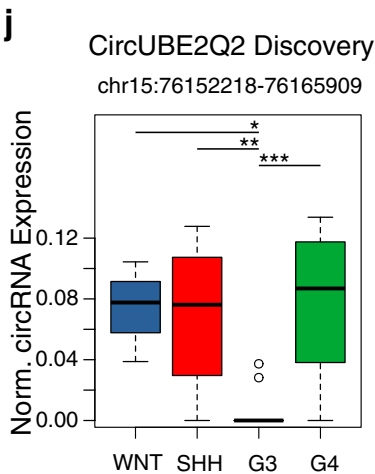

WNT SHH G3 G4 k

CircEXOC6B Discovery chr2:72945231-72960247

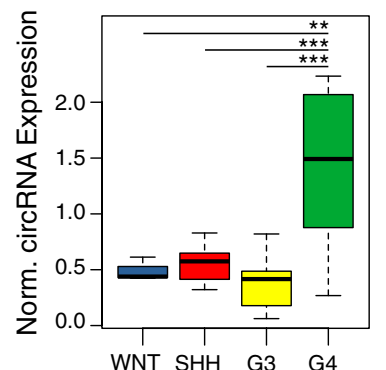

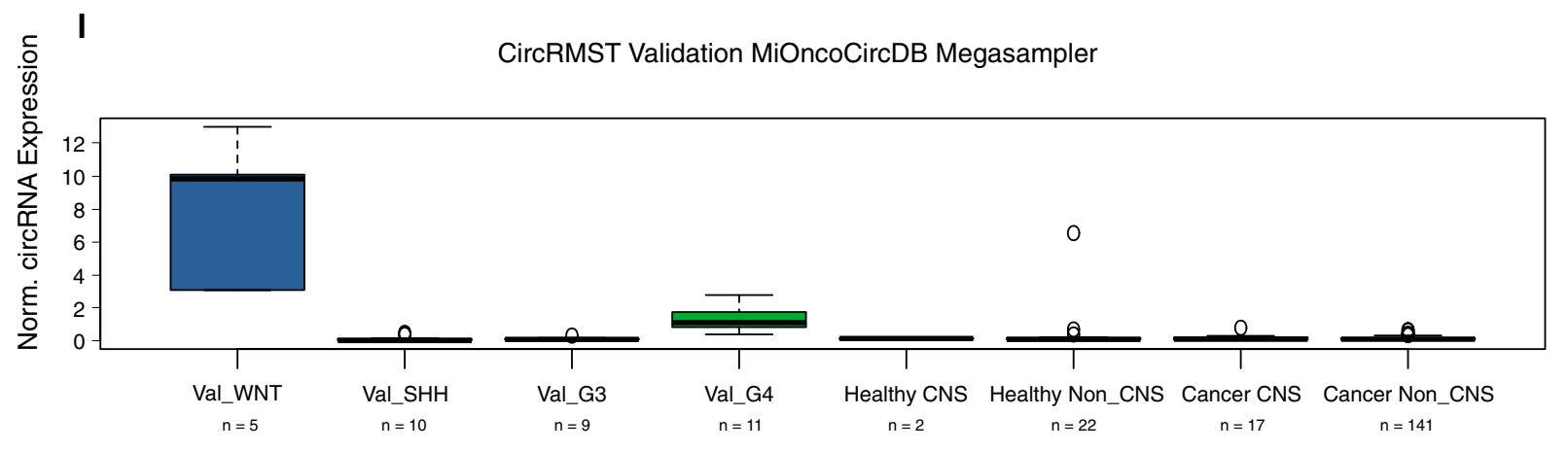


४Fig. 1 Circular RNA expression profiles define medulloblastoma groups. a Circular RNA (circRNA) signal across fetal brain controls (healthy, $n=12)$, discovery $(n=38)$ and validation medulloblastoma (MB) cohort $(n=35)$. b, c Heatmap of top 500 differentially expressed circRNAs in discovery (b) and in validation data set (c), hierarchical clustering by average Pearson dissimilarity, circRNA MB groups: WNT blue, SHH red, G3 yellow, G4 green. d Circular RNA signals across discovery data set similarity network fusion (SNF) MB groups. e Circular RNA signal across discovery data set (circRNA MB groups). f Circular RNA signal across validation data set (circRNA MB groups). $\mathbf{g}$ Medulloblastoma grouping according to [3] and circRNA data in discovery data set. MB36 was diagnosed as SHH-MB in the routine diagnostic setting. White square $=$ data not available. h-k. Boxplot of circRMST, circISPD, circUBE2Q2, and circEXOC6B in discovery data set with circRNA-based groups and genomic coordinates. I Megasampler with normalized circRMST expression in validation data set with the circRNA groups (Val_ WNT $=$ WNT validation data set; Val_SHH $=$ SHH validation data set; Val_G3 $=$ G3 validation data set; Val_G4 $=$ G4 validation data set) and MiOncoCircDB (four categories: healthy_CNS, healthy_non_ CNS, cancer_CNS, cancer_non_CNS, details see Tables S9 and S10). Tukey's HSD adjusted $p$ values: $* p<0.05 ; * * p<0.01 ; * * * p<0.001$

obtained from methylome, transcriptome, and proteome [3]) and using circRNA-based grouping in our discovery cohort (Fig. 1e) and in our validation cohort (Fig. 1f).

Using only circRNA expression profiles of the top 500 most differentially expressed circRNAs in our discovery cohort, we were able to reliably separate the four core groups almost as accurately as using SNF integrating multiomics data $(34 / 35=97.14 \%$ concordance; adjusted rand index $=0.91, p=0.001$; Fig. $1 \mathrm{~g}$ ).

To discern group-specific and subtype-specific circRNA biomarkers, we performed differential expression analysis of the circRNA-defined groups (Fig. 1h-k, Figures S2a-e, S3a-d) and of DNA methylation-defined subtypes (Figure S4a-d, Table S4). Significant and consistent up- or downregulations of circRNAs were identified in both cohorts for WNT ( $n=81$; Table S5, Figs. 1h, S2a, S3a), SHH $(n=7$; Table S6, Figs. 1i, S2b, S3b), and Group $4(n=13$; Table S7, Figs. 1k, S2d, S3d), while Group 3-specific circRNAs were not consistently detected (Table S8, Figs. 1j, S2c, S3c). Notably, a circRNA derived from the rhabdomyosarcoma 2-associated transcript (RMST) locus (chr12: 97886238-97954825) was aberrantly overexpressed in WNT medulloblastomas compared to the other groups $(p<0.001$; Figs. 1h, S2a). RMST is a well-known long non-coding RNA, which is exclusively expressed in brain tissue [6], and mostly present in circular isoforms [4]. Using a published cohort MiOncoCircDB consisting of circRNAs across over 2000 cancer samples [10], we validated circRMST as a highly reproducible and specific biomarker for WNT medulloblastoma (Fig. 1I).

In conclusion, we demonstrate a powerful and reliable method for molecular classification using our pipeline circs without the necessity to include additional information layers. Thus, circRNA-based biomarkers may help to improve diagnostic and therapeutic approaches in this highly aggressive disease.

Supplementary Information The online version contains supplementary material available at https://doi.org/10.1007/ s00401-021-02306-2.

Acknowledgements We thank especially Prof. Gunnar Klau for his bioinformatic support and mentorship. Furthermore, we would like to thank the tumor bank at the Necker Hospital. This work was supported by grants from the Deutsche Forschungsgemeinschaft (RE 2857/2-1 M.R., RE938/4-1, G.R., KFO 337, M.R., D. P.), JoséCarreras foundation (DJCLS 21R/2019, U.F., M.R.), Elterninitiative Kinderkrebsklinik e.V. (M.R., N.Q.), "Förderverein Löwenstern" to support children with cancer at Düsseldorf University Clinic (A.B), the Gert-und-Susanna-Mayer foundation (M.R.,J.B), Forschungskommission Heinrich Heine University (M.R., N.Q.), the German Cancer Aid (Deutsche Krebshilfe, grant no. 111537, G.R.), 'Courir pour la Vie, Courir pour Curie' and his president Dominique Ancelin, "Etoile de Martin" association, ARNP (association pour la recherche en neurochirurgie pédiatrique à necker), ITMO Cancer AVIESAN (National Alliance for Life Sciences and Health), within the framework of the Plan Cancer 2014-2019 and convention "2018, Non-coding RNA in cancerology: fundamental to translational (18CN039-00)", CNRS/INSERM and Institut Curie (O.A.).

Funding Open Access funding enabled and organized by Projekt DEAL.

Open Access This article is licensed under a Creative Commons Attribution 4.0 International License, which permits use, sharing, adaptation, distribution and reproduction in any medium or format, as long as you give appropriate credit to the original author(s) and the source, provide a link to the Creative Commons licence, and indicate if changes were made. The images or other third party material in this article are included in the article's Creative Commons licence, unless indicated otherwise in a credit line to the material. If material is not included in the article's Creative Commons licence and your intended use is not permitted by statutory regulation or exceeds the permitted use, you will need to obtain permission directly from the copyright holder. To view a copy of this licence, visit http://creativecommons.org/licenses/by/4.0/.

\section{References}

1. Cavalli FMG, Remke M, Rampasek L et al (2017) Intertumoral heterogeneity within medulloblastoma subgroups. Cancer Cell 31:737-754. https://doi.org/10.1016/j.ccell.2017.05.005 (e6)

2. Cheng J, Metge F, Dieterich C (2016) Specific identification and quantification of circular RNAs from sequencing data. Bioinformatics 32:1094-1096. https://doi.org/10.1093/bioinformatics/ btv656

3. Forget A, Martignetti L, Puget S et al (2018) Aberrant ERBB4SRC signaling as a hallmark of Group 4 medulloblastoma revealed by integrative phosphoproteomic profiling. Cancer Cell 34:379-395.e7. https://doi.org/10.1016/j.ccell.2018.08.002

4. Izuogu OG, Alhasan AA, Mellough C et al (2018) Analysis of human ES cell differentiation establishes that the dominant isoforms of the lncRNAs RMST and FIRRE are circular. BMC Genomics. https://doi.org/10.1186/s12864-018-4660-7

5. Memczak S, Jens M, Elefsinioti A et al (2013) Circular RNAs are a large class of animal RNAs with regulatory potency. Nature 495:333-338. https://doi.org/10.1038/nature11928 
6. Ng S-Y, Bogu GK, Soh BS et al (2013) The long noncoding RNA RMST interacts with SOX2 to regulate neurogenesis. Mol Cell 51:349-359. https://doi.org/10.1016/j.molcel.2013.07.017

7. Northcott PA, Robinson GW, Kratz CP et al (2019) Medulloblastoma. Nat Rev Dis Primers. https://doi.org/10.1038/ s41572-019-0063-6

8. Northcott PA, Shih DJH, Peacock J et al (2012) Subgroup-specific structural variation across 1000 medulloblastoma genomes. Nature 488:49-56. https://doi.org/10.1038/nature11327

9. Ramaswamy V, Samuel N, Remke M (2014) Can miRNA-based real-time PCR be used to classify medulloblastomas? CNS Oncol 3:173-175. https://doi.org/10.2217/cns.14.14
10. Vo JN, Cieslik M, Zhang Y et al (2019) The landscape of circular RNA in cancer. Cell 176:869-881.e13. https://doi.org/10. 1016/j.cell.2018.12.021

11. Zhang X-O, Wang H-B, Zhang Y et al (2014) Complementary sequence-mediated exon circularization. Cell 159:134-147. https://doi.org/10.1016/j.cell.2014.09.001

Publisher's Note Springer Nature remains neutral with regard to jurisdictional claims in published maps and institutional affiliations. 